\title{
Are symmetric tidal streams possible with long-range dark-matter forces?
}

\author{
Michael Kesden \\ California Institute of Technology, MC 130-33, 1200 East California Boulevard, Pasadena, California 91125, USA
}

(Received 23 March 2009; published 28 October 2009)

\begin{abstract}
The unique dynamics of the tidal disruption of satellite galaxies is an extremely sensitive probe of longrange interactions between dark-matter particles. Dark-matter forces that are several percent the strength of gravity will lead to order unity changes in the ratio of the number of stars in the leading and trailing tidal streams of a satellite galaxy. The approximate symmetry of the stellar tidal streams of the Sagittarius dwarf galaxy would thus exclude attractive dark-matter forces greater than $10 \%$ the strength of gravity which would entirely eliminate the leading stream. However, recent simulations suggest that dark-matter forces $100 \%$ the strength of gravity could completely strip the stellar component of Sagittarius of its dark matter, allowing for the subsequent development of symmetric tidal streams. Here we argue that these simulations use inconsistent initial conditions corresponding to separate pure stellar and pure dark-matter satellites moving independently in the host galaxy's halo, rather than a single disrupting composite satellite as had been intended. A new simulation with different initial conditions, in particular a much more massive satellite galaxy, might demonstrate a scenario in which symmetric tidal streams develop in the presence of large dark-matter forces. This scenario must satisfy several highly restrictive criteria described in this paper.
\end{abstract}

DOI: 10.1103/PhysRevD.80.083530

\section{INTRODUCTION}

Understanding the nature of dark matter (DM) remains one of the most important outstanding problems in cosmology. If DM is a new fundamental particle, it may experience new nongravitational interactions that would manifest as a violation of the weak equivalence principle. Such DM forces should be observationally constrained on all scales where DM influences structure, from the subgalactic out to the Hubble radius. In important early work, Frieman and Gradwohl [1,2] recognized that DM forces would violate the equivalence principle, and considered cosmological constraints on DM forces of the form

$$
\mathbf{F}=-\frac{\beta G m^{2}}{r^{2}} e^{-r / \lambda}\left(1+\frac{r}{\lambda}\right) \hat{\mathbf{r}}
$$

where $G$ is Newton's constant, $m$ is the mass of a DM particle, $r$ is the separation between particles, $\lambda$ is a "screening length" beyond which the force is suppressed, and $\beta$ is a dimensionless parameter characterizing the relative strength of this force compared to gravity [3]. Observations at the time of Frieman and Gradwohl's work excluded DM forces with $\beta \gtrsim 1.3$ and $\lambda \gtrsim$ $\mathcal{O}(1 \mathrm{Mpc})$.

DM forces received renewed attention after it was realized that they were generically produced by a coupling to dark energy [8] and could arise naturally in string theory $[9,10]$. They also might agree better with observations than the standard cosmological constant plus cold dark matter $(\Lambda \mathrm{CDM})$ scenario of structure formation [4]. Kesden and Kamionkowski ([6,7], hereafter KK06) examined the effects of DM forces on tidally disrupting satellite galaxies, where the large difference between the mass $m_{\text {sat }}$ of the
PACS numbers: 98.80.-k, 95.35.+d, 98.56.Wm, 98.65.Fz

satellite galaxy and that of the host galaxy $M_{\text {host }}$ establishes a hierarchy in the relevant energy scales of the problem. Assuming that the screening length $\lambda \gtrsim d$, an attractive DM force increases the satellite's orbital energy per unit mass

$$
E_{\text {orb }} \simeq \frac{G M_{\text {host }}}{d}
$$

by a factor $\beta f_{\mathrm{DM} \text {,host }} f_{\mathrm{DM} \text {, sat }}$, where $d$ is the distance between the satellite and host galaxies and $f_{\mathrm{DM}}$ is the DM mass fraction in the satellite or host. A tidally disrupted star will end up in the leading (trailing) stream if its binding energy per unit mass is less (greater) than the typical energy

$$
E_{\mathrm{bin}} \simeq \frac{G m_{\mathrm{sat}}}{r},
$$

where $r$ is the radius of the satellite galaxy. Equating the change in orbital energy induced by DM forces to the binding energy at the tidal radius

$$
r_{\text {tid }} \simeq\left(\frac{m_{\text {sat }}}{M_{\text {host }}}\right)^{1 / 3} d,
$$

we find that DM forces will produce an order unity change in the ratio of the number of stars in the leading to those in the trailing tidal streams when

$$
\beta \gtrsim \frac{1}{f_{\mathrm{DM}, \text { host }} f_{\mathrm{DM}, \mathrm{sat}}}\left(\frac{m_{\mathrm{sat}}}{M_{\mathrm{host}}}\right)^{2 / 3} .
$$

KK06 applied their constraints to the Sagittarius (Sgr) dwarf galaxy, a Milky Way satellite approximately $d \simeq$ $17 \mathrm{kpc}$ from the Galactic center discovered in 1994 [11] after the publication of Frieman and Gradwohl's work. The 
Sgr dwarf has leading and trailing stellar tidal streams stretching hundreds of degrees across the sky that have been observed extensively by the Two-Micron All-Sky Survey (2MASS) [12] and Sloan Digital Sky Survey (SDSS) [13]. The proposed mass-to-light ratio $(M / L)_{\text {Sgr }}=$ $14-36(M / L)_{\odot}$ of the Sgr dwarf suggests that it is DM dominated, as is the Milky Way at the perigalactic and apogalactic distances of the Sgr orbit, about 15 and $60 \mathrm{kpc}$ respectively [14]. The current bound mass of the Sgr dwarf is $(2-5) \times 10^{8} M_{\odot}$, implying $m_{\text {sat }} / M_{\text {host }} \simeq 10^{-3}$. Inserting this into Eq. (5) along with $f_{\mathrm{DM}, \text { host }} \sim f_{\mathrm{DM} \text {, sat }} \sim 1$, we find that DM forces several percent the strength of gravity should induce order unity changes in the leading-to-trailing ratio of stars in the Sgr tidal streams. $N$-body simulations confirmed that $\beta \geqslant 0.1$ completely eliminated the leading tidal stream for a wide range of models of the satellite galaxy, host galaxy, and satellite orbit. KK06 thus conservatively excluded $\beta \geqslant 0.1$, as the Sgr tidal streams are observed to be roughly symmetric.

Keselman, Nusser, and Peebles ([5], hereafter KNP09) have proposed an alternative scenario in which observations of the Sgr dwarf could be consistent with $\beta \simeq 1 \mathrm{DM}$ forces. In this proposal, the differential acceleration experienced by stars and DM segregate them from each other well before the first simulated pericentric passage. The self-bound stellar remnant of Sgr is free of DM and thus immune to DM forces. It proceeds to develop symmetric tidal streams in the Galactic gravitational potential well. The absence of a Sgr DM halo leaves the remaining stars more vulnerable to tidal heating during pericentric passages. The resulting heightened velocity dispersion could be misinterpreted as evidence for DM if the virial theorem was misapplied to this highly nonvirialized system. KNP09 argues that just such a misinterpretation could explain the high observed mass-to-light ratio $(M / L)_{\text {Sgr }}=$ 14-36(M/L) $\odot$ of Sgr, which would otherwise be incompatible with the complete segregation of stars and DM required by $\beta \simeq 1$.

Unfortunately, the initial conditions used in the simulations presented in KNP09 are not appropriate for describing this alternative scenario for the formation of the Sgr tidal streams. These simulations begin with the satellite galaxy's stellar and DM distributions moving with the same velocity and centered about the same point in the host galaxy's halo. In the absence of DM forces, two distributions with the same location and velocity are guaranteed to be gravitationally bound to each other. However, DM forces change the orbital energy of the DM particles by an amount $\Delta E \simeq \beta f_{\mathrm{DM} \text {,host }} E_{\text {orb }}$ that for $\beta=1$ exceeds the energy $E_{\text {bin }}$ binding these particles to the satellite. KNP09 notes that DM forces induce "a nearly complete segregation of the stars and DM well before the first pericentric passage." For these initial conditions, the stars and DM are already widely separated in orbital energy before the simulations even begin, though they initially coincide in phase space. This point is dramatized by noting that if these simulations were run backwards in time, by symmetry the stars and DM would be segregated just as efficiently. These simulations actually describe the temporary coincidental convergence of two different satellites, one purely stellar and the other pure DM, moving independently on very different orbits in the host galaxy's halo.

Proper initial conditions would show that this scenario cannot produce a purely stellar satellite with symmetric tidal streams like the Sgr dwarf. The segregation of stars and DM seen in these simulations will happen on the very first approach of the satellite galaxy to the Galactic center, long before dynamical friction can put the satellite onto an orbit resembling that of the current Sgr dwarf. The satellite will have fallen in from distances greater than the host galaxy's virial radius, and having been accelerated by a $\beta=1 \mathrm{DM}$ force will be moving at a velocity well above the gravitational escape velocity of the system. Once segregated from their DM halo, the satellite stars will be moving on a hyperbolic orbit and will be ejected from the host galaxy after a single pericentric passage.

We have identified three conditions that must be satisfied for the KNP09 scenario for the formation of the Sgr tidal streams to be viable:

(1) The DM force must be strong enough that differential acceleration in the Galactic halo fully segregates the stars and DM prior to the formation of the tidal streams.

(2) The stellar density in the satellite core must be high enough for it to remain a bound object after being pulled free from its DM halo.

(3) The DM force must not accelerate the satellite beyond the gravitational escape velocity of the host galaxy before the satellite stars are pulled from their DM halo.

We will describe our models of the host and satellite galaxies in Sec. II. We will then determine whether these models satisfy the three conditions given above in Sec. III. We will find that while $\beta=1 \mathrm{DM}$ forces are more than strong enough to fulfill condition 1, they are too strong to satisfy condition 3. Star formation is also unlikely to be efficient enough in dwarf galaxies to yield stellar densities that satisfy condition 2 . A summary and some concluding remarks will be provided in Sec. IV.

\section{GALACTIC MODELS}

\section{A. Host galaxy}

As we shall see in Sec. III A, for $\beta \gtrsim 1$ stars will become unbound from the satellite DM halo at galactocentric distances $d$ much greater than the scale lengths of the Galactic bulge or disk. For convenience, we will therefore model both these components as point particles at the Galactic center of masses $M_{\text {bulge }}$ and $M_{\text {disk }}$ respectively. Following KNP09, we will adopt values of $M_{\text {bulge }}=3.4 \times 10^{10} M_{\odot}$ and $M_{\text {bulge }}=10^{11} M_{\odot}$ for these 
quantities. KNP09 uses the density profile

$$
\rho_{\mathrm{DM}, \mathrm{host}, \mathrm{KNP}}(d)=\frac{v_{\text {halo }}^{2}}{2 \pi G} \frac{d^{2}+3 b^{2}}{\left(d^{2}+b^{2}\right)^{2}}
$$

for the Galactic DM halo, with $v_{\text {halo }}=131.5 \mathrm{~km} / \mathrm{s}$ and $b=12 \mathrm{kpc}$. We will assume spherical symmetry for the both the host and satellite galaxies, implying that for each component $X$ a mass

$$
M_{X}(d)=4 \pi \int_{0}^{d} r^{2} \rho_{X}(r) d r
$$

is contained within a sphere of radius $d$. The DM profile of Eq. (6) is unsuitable for our purposes because $M_{\mathrm{DM} \text {,host }}(d)$ does not asymptote to a finite value at large $d$, implying that an escape velocity

$$
v_{\mathrm{esc}}(d)=\left(\frac{2 G M(d)}{d}\right)^{1 / 2}
$$

cannot be defined. We will instead use a Navarro-FrenkWhite (NFW) profile $[15,16]$

$$
\rho_{\mathrm{DM}}(d)=\frac{\rho_{0}}{(r / b)(1+r / b)^{2}},
$$

with the same scale radius $b=12 \mathrm{kpc}$ and the normalization $\rho_{0}$ chosen to contain the same Galactic DM mass within the initial apocentric distance $d_{\text {ap }}=80 \mathrm{kpc}$ simulated in KNP09. This implies a virial mass $M_{\mathrm{v}, \text { host }}=$ $1.05 \times 10^{12} M_{\odot}$, virial radius $d_{\mathrm{v}}=206 \mathrm{kpc}$, and concentration $c_{\mathrm{v}, \text { host }} \equiv d_{\mathrm{v}} / b=17.2$, all very reasonable values for describing the Milky Way halo.

\section{B. Satellite galaxy}

Following the conventions of KNP09, we will model the stellar component of the satellite galaxy with a modified Hubble profile [17]

$$
\rho_{*, \mathrm{sat}}(r)=\rho_{1}\left(1+\frac{r^{2}}{r_{c}^{2}}\right)^{-3 / 2},
$$

where $r_{c}=0.55 \mathrm{kpc}$ and $\rho_{1}$ is chosen so that so that a stellar mass $M_{* \text {,sat }}\left(r_{t}\right)=3.0 \times 10^{8} M_{\odot}$ is contained within the tidal radius $r_{t}=1.67 \mathrm{kpc}$. Unlike KNP09 we will assume that the stellar density drops to zero outside $r_{t}$ as our criteria will be applied to the satellite core. The satellite galaxy's DM halo will also use the NFW profile of Eq. (9), but with a scale radius $r_{s}=3.7 \mathrm{kpc}$, virial radius $r_{v}=$ $18.65 \mathrm{kpc}$, and virial mass $M_{\mathrm{v}, \text { sat }}=1.5 \times 10^{9} M_{\odot}$. We will assume for simplicity that the DM density vanishes outside $r_{v}$, as the DM at large radii is largely irrelevant. This choice of parameters implies

$$
\frac{M_{* \text {, sat }}\left(r_{t}\right)}{M_{\mathrm{v}, \text { sat }}} \simeq \frac{\Omega_{b}}{\Omega_{\mathrm{DM}}} \simeq 0.2,
$$

and therefore that star formation is $100 \%$ efficient in satellite galaxies. This assumption is difficult to reconcile with theoretical arguments suggesting that feedback and reionization suppress star formation in low-mass halos [18], but we will consider it as a starting point.

\section{CONDITIONS FOR SYMMETRIC TIDAL STREAMS}

The satellite and host galaxies described in Sec. II must satisfy three distinct constraints if their interaction is to produce a tidally disrupting system with symmetric tidal streams in the presence of a $\beta=1 \mathrm{DM}$ force. We will identify and describe these three constraints in the three subsections below.

\section{A. DM segregation}

KK06 showed that DM-dominated satellite galaxies will develop asymmetric tidal streams in the presence of DM forces. KNP09 argues that this can be avoided if nearly all of the DM is removed from the satellite galaxy before its stellar tidal streams begin to form. As the satellite galaxy descends deeper into the potential well of the host galaxy, both stars and DM will be stripped away by conventional gravitational tidal forces. This occurs because parts of the satellite at different distances from the center of the host galaxy experience different gravitational accelerations. The weak equivalence principle implies that at the same position, all objects experience the same gravitational acceleration. However, DM forces violate the equivalence principle and individual stars, individual DM particles, and the partly stellar, partly DM satellite galaxy will experience three different accelerations towards the host galaxy's DM halo. In a reference frame freely falling with the satellite as a whole, the individual stars will experience a relative acceleration

$$
a_{* \text { host }}(d)=\frac{G \beta f_{\mathrm{DM}, \mathrm{sat}} M_{\mathrm{DM}, \mathrm{host}}(d)}{d^{2}},
$$

while the DM particles experience a relative acceleration

$$
a_{\mathrm{DM}-\mathrm{host}}(d)=\frac{G \beta\left(1-f_{\mathrm{DM}, \mathrm{sat}}\right) M_{\mathrm{DM}, \mathrm{host}}(d)}{d^{2}} .
$$

In the absence of DM forces $(\beta=0)$, these relative accelerations vanish as required by the equivalence principle. Furthermore, stars will experience no acceleration relative to a purely stellar $\left(f_{\mathrm{DM} \text {,sat }}=0\right)$ satellite galaxy and DM particles will similarly experience no acceleration relative a purely $\mathrm{DM}\left(f_{\mathrm{DM} \text {,sat }}=1\right)$ satellite.

Tidal forces, supplemented by these relative accelerations, will succeed in disrupting the satellite galaxy if they are stronger than the attractive forces that act to keep stars and DM bound to the satellite. For stars this restoring acceleration is simply the gravitational attraction

$$
a_{*-\mathrm{sat}}(r)=\frac{G\left[M_{* \text {,sat }}(r)+M_{\mathrm{DM}, \mathrm{sat}}(r)\right]}{r^{2}}
$$


between the stars and the satellite, while for DM particles this gravitational restoring force is supplemented by the DM attraction to the satellite's DM halo

$$
a_{\mathrm{DM}-\mathrm{sat}}(r)=\frac{G\left[M_{*, \mathrm{sat}}(r)+(1+\beta) M_{\mathrm{DM}, \mathrm{sat}}(r)\right]}{r^{2}} .
$$

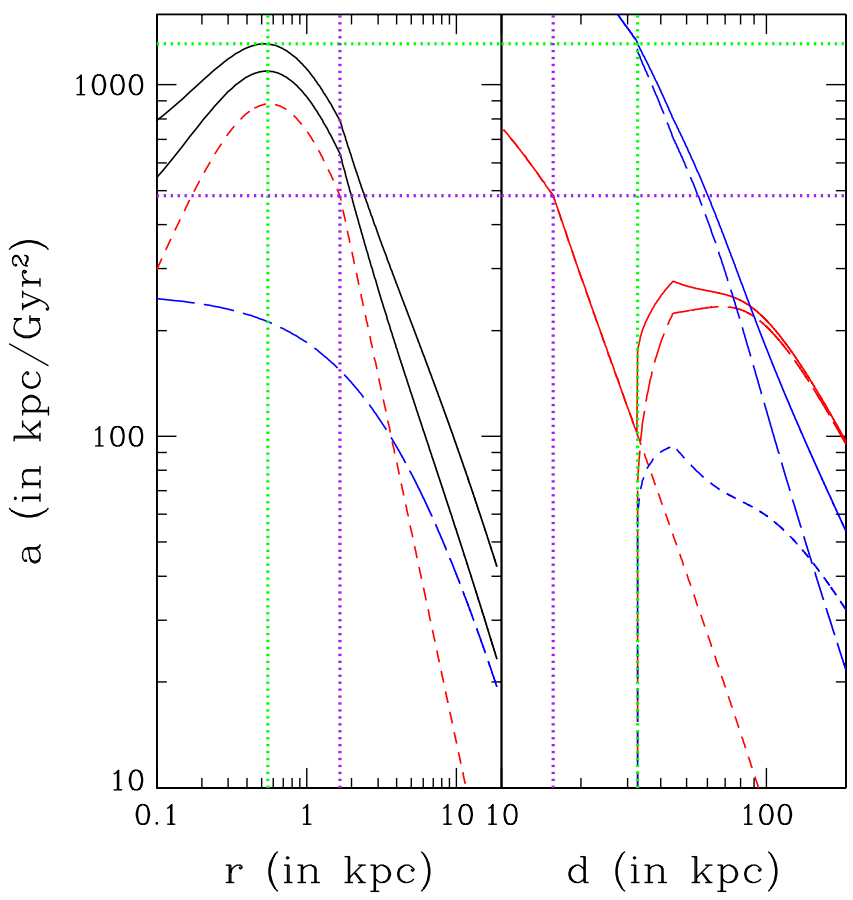

FIG. 1 (color online). The accelerations $a$ experienced by stars and DM particles a distance $r$ from the center of the satellite galaxy and $d$ from the center of the host galaxy. Left-hand panel: The lower solid black curve shows the gravitational attraction $a_{*-\text { sat }}$ between stars and the satellite galaxy. The short-dashed red and long-dashed blue curves show the contributions of stars and DM respectively to this gravitational attraction. The upper solid black curve shows the acceleration $a_{\mathrm{DM} \text {-sat }}$ between DM particles and the satellite; the DM contribution has been doubled relative to $a_{* \text {-sat }}$ for $1+\beta=2$. Right-hand panel: The short-dashed blue curve shows the tidal acceleration at the edge of the satellite's DM halo, the long-dashed blue curve shows the relative acceleration $a_{\mathrm{DM} \text {-host }}$, and the solid blue curve shows the sum of these two accelerations. The three red curves show the corresponding quantities for the stars. The satellite is fully stripped of DM when it first reaches a distance $d_{\text {seg }}=32.7 \mathrm{kpc}$ from the center of the host galaxy as shown by the vertical dotted green line in the right-hand panel. At this distance, the total relative acceleration experienced by DM particles (solid blue curve in right-hand panel) equals the maximum of the restoring acceleration (upper solid black curve in left-hand panel) which is located near the satellite's stellar core radius $r_{c}=0.55 \mathrm{kpc}$ (vertical dotted green line in left-hand panel). Tidal disruption of the stars does not begin until $d_{\text {tid }}=15.7 \mathrm{kpc}$ (vertical dotted purple line in righthand panel) when the total relative acceleration experienced by stars (solid red curve in right-hand panel) equals the restoring acceleration for stars at the stellar tidal radius $r_{t}=1.67 \mathrm{kpc}$ (vertical dotted purple line in left-hand panel).
These accelerations are shown in Fig. 1 for the galactic models described in Sec. II with a $\beta=1 \mathrm{DM}$ force. The many curves in this figure are described in its long caption. Disruption of the satellite's extended DM halo has already begun even at the host galaxy's virial radius $d_{v}=206 \mathrm{kpc}$. This can be seen by observing that the upper solid black curve $a_{\mathrm{DM} \text {-sat }}$ in the left-hand panel intersects the right boundary below where the solid blue curve $a_{\mathrm{DM} \text {-sat }}$ in the right-hand panel intersects the right boundary. As the satellite falls inwards, the radius $r$ and mass $M$ of its DM halo decrease as shown by the long-dashed blue curves in Fig. 2. Although the tidal forces initially dominate the disruption, the relative acceleration $a_{\mathrm{DM} \text {-host }}$ exceeds the tidal acceleration for $d<147.6 \mathrm{kpc}$ where the long-dashed blue curve crosses the short-dashed blue curve in the righthand panel of Fig. 1. Disruption of the satellite's DM halo continues until $d_{\text {seg }}=32.7 \mathrm{kpc}$, when the total relative acceleration of the DM exceeds the maximum of the restoring acceleration $a_{\mathrm{DM}-\mathrm{sat}}$. At this distance all the remaining DM mass $M_{\mathrm{DM} \text {, sat }}\left(r_{c}\right)=1.6 \times 10^{7} M_{\odot}$ interior to the stellar core radius $r_{c}=0.55 \mathrm{kpc}$ where this maximum occurs is disrupted en masse. This occurs because

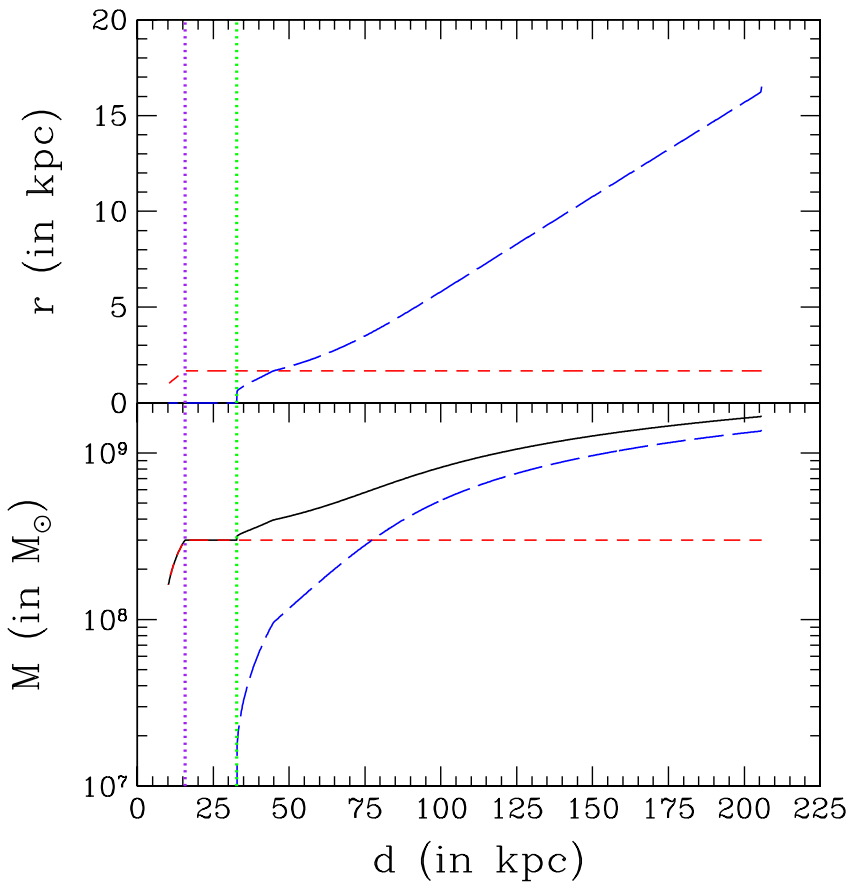

FIG. 2 (color online). Upper panel: The radius $r$ of satellite's DM halo (long-dashed blue curve) and stellar distribution (shortdashed red curve) as the satellite gets within a distance $d$ of the host galaxy. Lower panel: The mass $M$ of the satellite galaxy (solid black curve), its DM halo (long-dashed blue curve), and stellar distribution (short-dashed red curve) at a distance $d$ from the host galaxy. The vertical dotted green curve shows the distance $d_{\text {seg }}=32.7 \mathrm{kpc}$ at which the stars and DM are fully segregated, while the vertical dotted purple curve shows the distance $d_{\text {tid }}=15.7 \mathrm{kpc}$ at which tidal disruption of the stellar component begins. 
$a_{\mathrm{DM}-\mathrm{sat}}(r)$ is not a monotonically decreasing function of $r$ as a result of the cored stellar profile $\rho_{* \text { sat }}(r)$ of Eq. (10). The sharp drop in $M_{\mathrm{DM} \text {, sat }}$ leads to corresponding sharp drops in $f_{\mathrm{DM} \text {,sat }}$ and $a_{*-\text { host }}$ in Eq. (12), explaining the cuspy nature of the total relative acceleration for stars shown by the solid red curve in the right-hand panel of Fig. 1. This cored stellar profile is very conducive to the scenario of KNP09, as a cuspy profile would have had a greater central restoring acceleration $a_{\mathrm{DM}-\mathrm{sat}}(0)$ and might have retained DM until stellar tidal disruption commenced. The satellite galaxy, now entirely free of its DM halo, remains undisturbed until gravitational tidal forces on their own can disrupt it in the standard manner. This occurs at a distance $d_{\text {tid }}=15.7 \mathrm{kpc}$ from the host galaxy where the tidal forces exceed the restoring acceleration $a_{*-\text { sat }}\left(r_{t}\right)$ at the satellite's tidal radius $r_{t}=1.67 \mathrm{kpc}$.

Simulation S2 of KNP09 was designed to illustrate how symmetric tidal streams resembling those of the Sgr dwarf could come about even in the presence of $\beta=1 \mathrm{DM}$ forces. It begins with the satellite at an apocentric distance of $d_{\text {ap }}=84.8 \mathrm{kpc}$. Although the pericentric distance is not provided explicitly, if the streams are to resemble those of Sgr it must be close to Sgr's estimated pericentric distance of $d_{\mathrm{pe}} \simeq 14 \mathrm{kpc}$ [14]. For this choice of an initial orbit we have

$$
d_{\mathrm{pe}}<d_{\text {tid }}<d_{\text {seg }}<d_{\text {ap }}
$$

which demonstrates one of the concerns with this simulation. Although the satellite begins the simulation with its DM halo intact out to the virial radius $r_{v}$, this extended halo should have been disrupted at much greater distances from the host galaxy. Since $d_{\mathrm{pe}}<d_{\text {seg }}$, the satellite should not have any DM halo at all. These initial conditions represent a temporary association of stars and DM moving independently in the Galactic halo, like ships passing in the night. To properly simulate this scenario, the satellite's orbit must evolve from that on which the DM was fully segregated $\left(d_{\mathrm{pe}}=d_{\mathrm{seg}}\right)$ to the current orbit needed to produce the observed stellar tidal streams.

Dynamical friction drives this orbital evolution, but was neglected in KNP09 since the streams develop on a few orbital times $t_{\text {orb }}$ and according to [17] the dynamicalfriction time

$$
t_{\mathrm{DF}} \sim\left(\frac{M_{\mathrm{host}}}{m_{\mathrm{sat}}}\right) t_{\mathrm{orb}} \simeq 10^{3} t_{\mathrm{orb}}
$$

is much longer than this for the Sgr-Milky Way system. In response to this paper, KNP09 performed a new simulation with a "live" DM halo for the host galaxy that allowed for dynamical friction and a more massive satellite $m_{\text {sat }} / M_{\text {host }} \simeq 0.1$ that would reduce the dynamical-friction time $t_{\mathrm{DF}}$. The satellite also began on an orbit with a greater apocentric distance $d_{\mathrm{ap}}=250 \mathrm{kpc}$ to allow for orbital evolution. A detailed analysis of this new simulation is beyond the scope of this paper, but it should be examined whether this live halo can adequately describe the dynamical friction given that the final satellite mass is $m_{\text {sat }} \leqslant 4 \times$ $10^{8} M_{\odot}$ and the individual $N$-body particles of the host galaxy's DM halo have masses of $10^{8} M_{\odot}$.

The segregation distance $d_{\text {seg }}=32.7 \mathrm{kpc}$ for the Sgr dwarf is comparable to the current distances of the newly discovered ultrafaint SDSS dwarf galaxies Segue 1, Ursa Major II, Willman 1, and Coma, all of which lie within $50 \mathrm{kpc}$ of the Galactic center. These new ultrafaint dwarf galaxies have a wide range of luminosities $\left(10^{2} \lesssim L / L_{\odot} \lesssim\right.$ $\left.10^{7}\right)$, yet share a common central density $M(r=$ $0.3 \mathrm{kpc}) \simeq 10^{7} M_{\odot}$ [19]. Simulations show that tidal heating cannot inflate the velocity dispersions of these satellites enough to eliminate the need for massive DM halos [20]. The satellites less luminous than Sgr will thus have higher DM fractions $f_{\mathrm{DM} \text {,sat }}$ and correspondingly higher relative accelerations $a_{* \text {-host }}$ for stars according to Eq. (12). The common central density however implies that the restoring acceleration $a_{*-\text { sat }}$ for the stars will remain largely independent of satellite luminosity. The stars should therefore be fully stripped from their DM halos at greater distances than $d_{\text {seg }}=32.7 \mathrm{kpc}$ for the more massive Sgr dwarf. This is not observed for Segue 1, the closest of the new dwarf satellites, whose galactocentric distance of $28 \mathrm{kpc}$ [21] is well within its predicted $d_{\text {seg }}$ for $\beta=1$. Although the ultrafaint dwarf satellites appear to place even tighter constraints on DM forces, if we restrict our attention to Sgr we see that a $\beta=1 \mathrm{DM}$ force is strong enough to segregate stars and DM in Sgr at distances $d_{\text {seg }}>d_{\text {tid }}$ before the stellar tidal streams begin to form. The first of our three criteria for Sgr to form symmetric tidal streams as proposed in KNP09 is thus satisfied.

\section{B. Intact stellar core}

Even if DM forces are strong enough to fully segregate a satellite galaxy's stars from their DM halo, the segregated stars may not survive as a self-bound object after being pulled free. The survival of an intact stellar core is essential to the scenario proposed in KNP09, as it is this remnant that subsequently develops the symmetric tidal streams observed today. The stellar core is threatened with destruction at three distinct stages:

(1) The satellite galaxy may be stripped of all its stars before the stars and DM are fully segregated.

(2) The stellar component of the satellite may never have been self-bound, but merely held together by the gravity of the satellite's DM halo.

(3) Even if the stars are initially self-bound, they may be tidally disrupted by the satellite's DM halo as it is segregated.

We will explore each of these threats in turn in the remainder of this subsection. 


\section{Competitive segregation}

We saw in the previous subsection that stars will be stripped from the satellite when the sum of the gravitational tidal acceleration and the relative acceleration $a_{* \text {-host }}$ exceed the restoring acceleration $a_{*-\text { sat }}$, while DM particles will be stripped when the same inequality is reached between $a_{\mathrm{DM} \text {-host }}$ and $a_{\mathrm{DM} \text {-sat }}$. This establishes a competition between stars and DM: if the DM profile is sufficiently cuspy or the satellite is sufficiently DM-dominated, there may not be a stellar remnant left when the satellite finally reaches $d_{\text {seg }}$. Returning to the accelerations plotted in Fig. 1, we see that at large $d$ the total relative acceleration for stars shown by the solid red curve in the right-hand panel was above the total relative acceleration for DM shown by the solid blue curve. If the satellite's DM halo had been more massive or less extended compared to the stellar component, $f_{\mathrm{DM}, \text { sat }}$ would have remained close to unity and the total relative acceleration for stars could have exceeded the maximum of the lower black curve in the lefthand panel before this happened for the DM at $d_{\text {seg }}$. In this case, all of the stars exterior to this maximum located at $r \simeq r_{c}$ would have been disrupted into asymmetric tidal streams. A small stellar remnant would remain because all of the stars interior to $r_{c}$ would have been stripped en masse as was the case for DM in our model of the Sgr dwarf in the previous subsection. If the restoring acceleration $a_{*-s a t}$ had been monotonically decreasing no bound stellar remnant would survive.

Though we have estimates of the total mass and mass-tolight ratio of Sgr, the stellar and DM density profiles themselves are very uncertain. It is worth exploring more generally which profiles will allow an intact stellar core to be segregated from its DM halo. A general DM density profile will scale as $\rho_{\mathrm{DM}}(r) \propto r^{-\alpha}$ at small $r$; the NFW profile of Eq. (9) has $\alpha=1$. The profile $\alpha=1$ is special in that the resulting acceleration $a_{\mathrm{DM}}(r)=G M_{\mathrm{DM}}(r) / r^{2} \propto$ $r^{1-\alpha}$ will be independent of $r$. DM halos with $\alpha>1$ yield accelerations that diverge at $r=0$ and monotonically decrease with $r$. Density profiles with $\alpha<1$ will produce accelerations that increase with $r$, before ultimately turning over at larger $r$ since the halo must have finite mass.

Of these three possibilities, the $\alpha=1$ profile is the most conducive to leaving a bound stellar remnant behind. DM halos with $\alpha>1$ will hold onto their innermost stars so tightly that $d_{\text {seg }}=0$ will always be less than $d_{\text {tid }}$ at which stellar tidal disruption begins. Simulation S3 of KNP09, though still employing an NFW profile for the satellite halo, has an enhanced central concentration that mimics this possibility. When this simulation is run, the majority of stars are indeed stripped from the satellite halo while the inner stellar core remains bound. Satellites with $\alpha<1$ will have accelerations that vanish at $r=0$, so even small differential accelerations $a_{\text {dif }}$ will displace the stars from the center of their DM halo. As the satellite falls inwards, $a_{*-\text { host }}$ increases and the stars march up the DM potential well to ever larger accelerations $a_{*-\text { sat }}$ before breaking free from the satellite when they reach the global maximum. However, because the satellite stellar distribution has a finite spatial extent, its outer edges will spill over the global maximum before the center arrives there. This may cause additional stars to be stripped from the satellite before the eventual bound stellar remnant itself breaks free.

If $\alpha$ was truly unity for an NFW profile, not just for $r \ll$ $b$, the acceleration $a_{\mathrm{DM}}(r)$ depicted by the long-dashed blue curve in the left-hand panel of Fig. 1 would be flat. If star formation was much less efficient as seems to be the case for less massive dwarf satellites, the DM contribution would dominate in $a_{*-\text { sat }}$ and the lower solid black curve would be flat as well. In this case, the total relative acceleration would rise above $a_{*-\text { sat }}(r)$ simultaneously over a wide range in $r$. The stars would slide out of their DM halo like "an egg sliding off a frying pan" [22], and the stars would be more likely to remain self-bound. In the competition to remain bound to the satellite galaxy, stars are more likely to defeat DM if stellar densities are high and centrally concentrated, while the DM density profile's logarithmic slope scales as $\alpha=1$ over a wide range.

\section{Self-boundedness}

After being segregated from its DM halo, the stellar remnant may no longer have enough self-gravity to remain bound. Unbound stars in the host galaxy's halo would develop into an orphan stream lacking a clear progenitor. One such system, apply dubbed the "Orphan Stream," was discovered serendipitously by SDSS [23]. This inspired simulation S4 in KNP09 which demonstrates just how readily such systems can be produced following the segregation of stars and DM by large DM forces. The possibility of making an orphan stream is a problem for efforts to explain the Sgr dwarf, which has a well-defined bound core identified by a cusp in the $M$-giant density distribution spatially coincident with the globular cluster M54 [12]. This bound core serves as a marker to distinguish the leading and trailing tidal streams of Sgr, essential to the proposal of KK06 to constrain DM forces by comparing the stars in each group.

Whether the satellite stars remain bound after their DM halo is removed depends on their distribution function $f_{* \text {,sat }}(\vec{r}, \vec{v})$. If the stars are segregated from the DM on a dynamical time that is shorter than the relaxation time on which their individual energies change, one can use an impulse approximation to determine which stars remain bound. Without the negative contribution of the DM halo's gravitational potential, some portions of phase space that were previously bound will now have positive energies. The integral of $f_{* \text {, sat }}(\vec{r}, \vec{v})$ over this portion of phase space will determine the initial mass loss. Without these stars the satellite's potential well will become even shallower, and the stars will rearrange themselves with further mass loss likely. Unfortunately, many different distribution functions 
will yield the density profile of Eq. (10). Distinguishing between them requires knowledge of the anisotropy of the satellite's velocity dispersion, which is even more poorly constrained observationally than the density profile itself.

One can crudely estimate whether the satellite stars remain bound by applying the virial theorem to the initial system to determine its kinetic energy

$$
\begin{aligned}
K & =-\frac{1}{2} W_{i} \\
& =-\frac{1}{4} \int \rho_{*}(r)\left[\Phi_{*}(r)+\Phi_{\mathrm{DM}<r_{t}}(r)\right] d^{3} \vec{r} .
\end{aligned}
$$

Here $W_{i}$ is the initial gravitational potential energy of the stars, $\Phi_{*}(r)$ is the gravitational potential of the satellite stars, and $\Phi_{\mathrm{DM}<r_{t}}(r)$ is the gravitational potential sourced by DM located interior to $r_{t}$. Spherical symmetry implies that only the mass interior to $r_{t}$ can accelerate the stars and thus contribute to their kinetic energy $K$. If the DM halo is removed quickly enough, this kinetic energy $K$ will remain unchanged while the final potential energy will be

$$
W_{f}=\frac{1}{2} \int \rho_{*}(r) \Phi_{*}(r) d^{3} \vec{r}
$$

now that the DM halo has been removed. The total final energy $E_{f}$ can be found by summing Eqs. (18) and (19)

$$
\begin{aligned}
E_{f} & =K+W_{f} \\
& =\frac{1}{4} \int \rho_{*}(r)\left[\Phi_{*}(r)-\Phi_{\mathrm{DM}<r_{t}}(r)\right] d^{3} \vec{r} .
\end{aligned}
$$

If $E_{f}>0$, the satellite as a whole will be unbound, though a bound remnant may still remain if the unbound stars carry away enough energy. Figure 3 shows $\Phi_{*}$ and $\Phi_{\mathrm{DM}<r_{t}}$ for the galactic models described in Sec. II. The dot-dashed black curve shows that their difference, appearing in the integrand of Eq. (20), is negative for $r \leq r_{t}$. Since $\rho_{*}(r)$ is positive definite, $E_{f}$ must be negative implying that the stellar remnant after DM segregation remains self-bound. If star formation had been less efficient or the DM profile was cuspier, we could have had $\Phi_{*}>$ $\Phi_{\mathrm{DM}<r_{t}}$ for some $r$ which might have driven $E_{f}$ positive. Only star formation efficiencies approaching the theoretical maximum of Eq. (11) allow the possibility of leaving a bound stellar remnant behind after the stars are segregated from their DM halo.

\section{Tidal disruption}

Even if the stellar remnant of the satellite is self-bound when initially displaced from its DM halo, it may be tidally disrupted by the satellite DM halo's gravity before it escapes beyond the satellite virial radius $r_{v}$. This process is in some ways the reverse of traditional tidal disruption, in that the stars begin at the center $r=0$ of the satellite DM halo and are pulled to larger $r$ by the relative acceleration, rather than beginning at a large distance $d$ from the

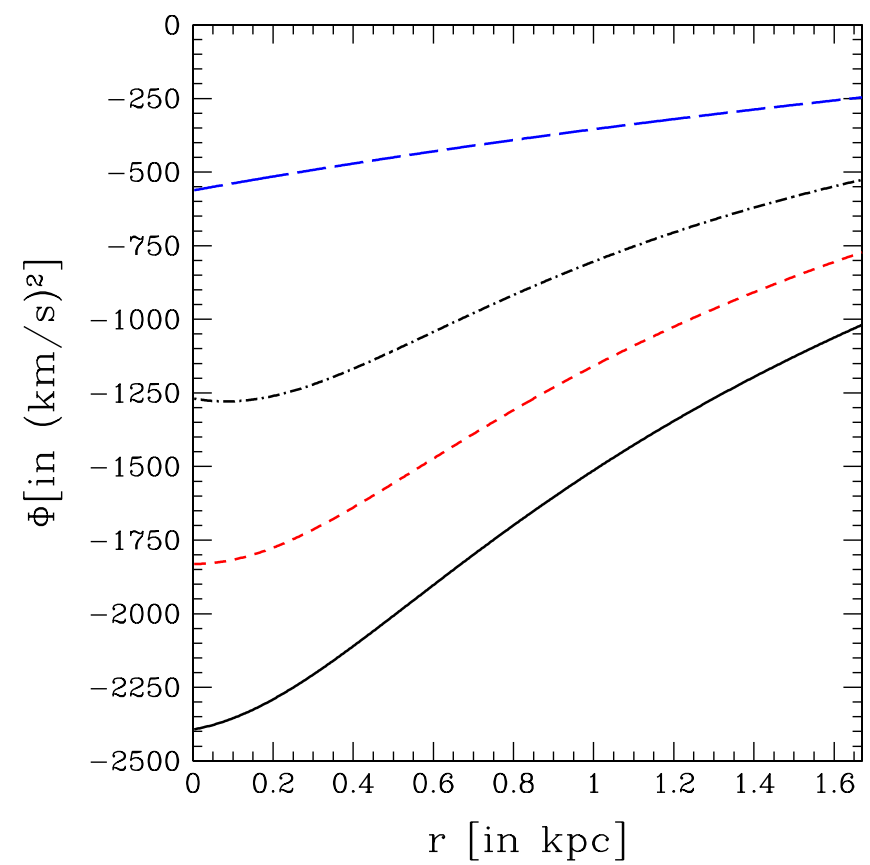

FIG. 3 (color online). Gravitational potentials $\Phi$ as a function of the distance $r$ from the center of the satellite galaxy. The short-dashed red curve is the potential $\Phi_{*}$ from stars, the longdashed blue curve is the potential $\Phi_{\mathrm{DM}<r_{t}}$ from DM interior to the tidal radius $r_{t}$, and the solid black curve is their sum. The dotdashed black curve is their difference $\Phi_{*}-\Phi_{\mathrm{DM}}$ whose stellarmass-weighted average, appearing in Eq. (20), determines whether the satellite stars remain bound after the DM halo has been removed.

host and falling inwards towards $d=0$. The tidal interactions between comparable-mass systems like the satellite stellar and DM distributions are extremely difficult to model analytically, necessitating the $\mathrm{N}$-body simulations used by both KK06 and KNP09. The best we can hope to do without such simulations is to compare the restoring acceleration at the edge of the stellar remnant, given by the stellar contribution to $a_{*-\text { sat }}\left(r_{t}\right)$, to the tidal acceleration sourced by the satellite's DM halo given by the DM contribution to $\left|a_{*-\text { sat }}(r)-a_{*-\text { sat }}\left(r+r_{t}\right)\right|$. The left-hand panel of Fig. 1 shows that the stellar contribution to $a_{*-\text { sat }}$ (shortdashed red curve) in the range $0 \leq r \leq r_{t}$ is greater than the DM contribution (long-dashed blue curve) over the entire range $0 \leq r \leq \infty$. This implies that the satellite DM halo cannot disrupt the stellar remnant during the segregation of the two components. As for the other two constraints considered in this section, this might not have been true for a cuspier DM profile or less massive stellar component of the satellite galaxy.

\section{Satellite bound to host galaxy}

The final condition that must be satisfied for the KNP09 scenario for the formation of symmetric tidal streams is that the satellite stars remain bound to the host galaxy after 
they are segregated from their DM halo. This is a significant concern, as even in the absence of DM forces the energy

$$
E_{\mathrm{tid}} \simeq \frac{\partial \Phi_{\mathrm{host}}}{\partial d} r_{\mathrm{tid}} \simeq\left(\frac{m_{\mathrm{sat}}}{M_{\mathrm{host}}}\right)^{1 / 3} E_{\mathrm{orb}}
$$

gained during tidal disruption can sometimes unbind stars in the trailing tidal stream from the host galaxy. This energy $E_{\text {tid }}$ is responsible for raising extended tidal tails in interacting galaxies, and has even been proposed as an explanation for the large fraction of hypervelocity stars with common travel times clustered in the direction of the constellation Leo [24]. The satellite galaxies in simulations $\mathrm{S} 1$ and S2 of KNP09 begin $\sim 80 \mathrm{kpc}$ from the host galaxy with velocities of $80 \mathrm{~km} / \mathrm{s}$, much less than the escape velocity $v_{\text {esc }}=333 \mathrm{~km} / \mathrm{s}$ at this distance. Dynamical friction over several orbits is assumed to have placed the satellite on this orbit, similar to that of the Sgr dwarf, before segregation occurs and the tidal streams begin to develop. However, we saw in Sec. III A that the stars will be segregated from their DM halo the first time the satellite gets with a distance $d_{\text {seg }}$ of the host galaxy. The satellite will be traveling at a greater velocity on this first approach and therefore its stars are more likely to become unbound from the host galaxy.

The secondary infall of subsequent material onto an already collapsed structure has been studied extensively in a cosmological context [25-27]. At early times this material recedes with the Hubble expansion, before reaching a maximum turnaround distance $d_{\mathrm{ta}}$ and falling back onto its host galaxy. Models of secondary infall can be used to predict the orbits of satellite galaxies [28], and comparison with observations suggest $d_{\mathrm{ta}} \simeq 1 \mathrm{Mpc}$ for the Milky Way [29]. Surviving satellites like the Sgr dwarf are biased towards large turnaround distances $d_{\mathrm{ta}}$ compared to disrupted satellites that form the Galactic stellar halo [30].

In Fig. 4 we show the velocities acquired by stars, DM particles, and the satellite as they freely fall towards the host galaxy on radial orbits from the turnaround distance. These estimated velocities are conservative, as the tangential velocities must be added in quadrature to determine the true total velocity. The velocities are calculated by assuming conservation of energy

$$
\frac{1}{2} v_{\mathrm{ff}}^{2}(d)=\int_{d}^{d_{\mathrm{ta}}} a_{\mathrm{ff}}(x) d x
$$

where the different free-fall accelerations $a_{\mathrm{ff}}$ for the stars, DM particles, and satellite are

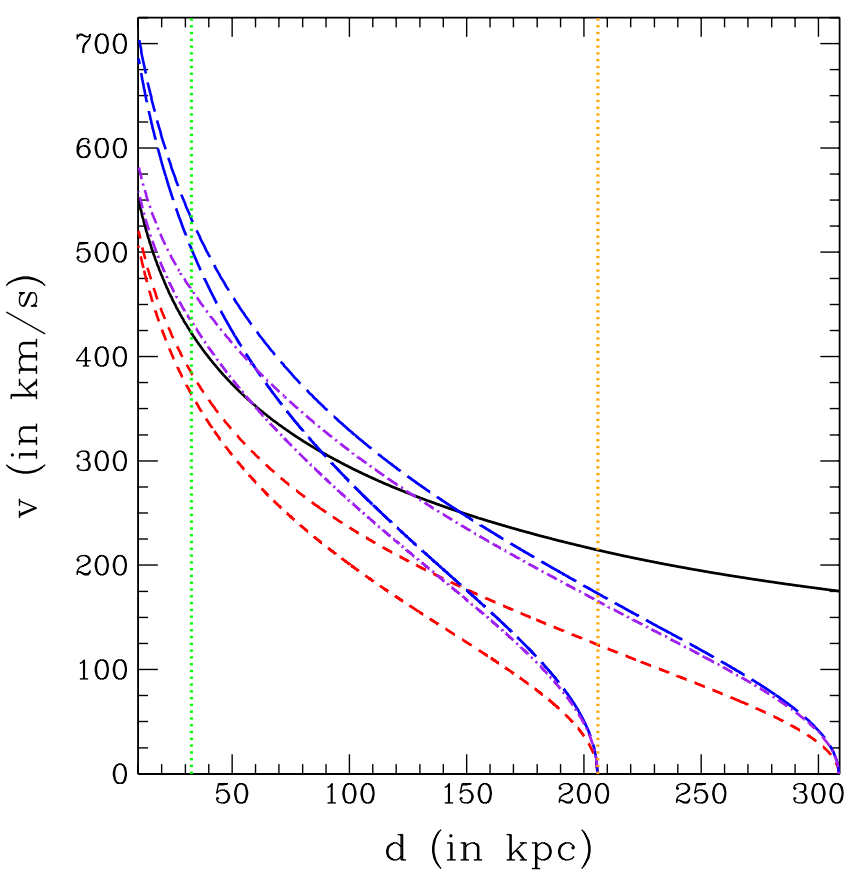

FIG. 4 (color online). Velocities $v$ of infalling stars, DM particles, and the satellite galaxy as a function of their distance $d$ from the host galaxy. These velocities are for radial infalls beginning at rest from turnaround distances $d_{\mathrm{ta}}$ equal to the host galaxy's virial radius $d_{v}=206 \mathrm{kpc}$ and $1.5 d_{v}$. The short-dashed red curves correspond to freely falling stars, the long-dashed blue curves to DM particles, and the dot-dashed purple curves to the satellite galaxy which is partly stellar and partly DM. The solid black curve shows the gravitational escape velocity $v_{\text {esc }}$. The dotted vertical orange line shows the host virial radius $d_{v}$, while the dotted vertical green line shows the segregation distance $d_{\text {seg }}$.

$$
\begin{aligned}
a_{*-\mathrm{ff}}(d) & =\frac{G\left[M_{*, \mathrm{host}}+M_{\mathrm{DM}, \mathrm{host}}(d)\right]}{d^{2}} \\
a_{\mathrm{DM}-\mathrm{ff}}(d) & =\frac{G\left[M_{*, \mathrm{host}}+(1+\beta) M_{\mathrm{DM}, \mathrm{host}}(d)\right]}{d^{2}} \\
a_{\mathrm{sat}-\mathrm{ff}}(d) & =\frac{G\left[M_{* \text { host }}+\left(1+\beta f_{\mathrm{DM}, \mathrm{sat}}\right) M_{\mathrm{DM}, \mathrm{host}}(d)\right]}{d^{2}} .
\end{aligned}
$$

The DM fraction $f_{\mathrm{DM} \text {,sat }}$ in Eq. (23c) is calculated selfconsistently as the ratio of the satellite's DM and total masses, given respectively by the long-dashed blue and solid black curves in the bottom panel of Fig. 2. Note that the difference $\left|a_{\text {sat-ff }}-a_{*-\text { ff }}\right|$ of these free-fall accelerations gives the relative acceleration $a_{*-\text { host }}$ appearing in Eq. (12). The difference $\left|a_{\mathrm{DM}-\mathrm{ff}}-a_{\mathrm{sat}-\mathrm{ff}}\right|$ similarly gives the relative acceleration $a_{\text {DM-host }}$ in Eq. (13).

The attractive DM force proportional to $\beta$ in Eqs. (23b) and $(23 \mathrm{c})$ accelerates the DM particles and satellite to higher velocities than the stars can attain by gravity alone. This can be seen in Fig. 4 where the DM velocities $v_{\text {DM-ff }}$ given by the long-dashed blue curves and the satellite 
velocities $v_{\text {sat-ff given by the dot-dashed purple curves }}$ exceed the stellar velocities $v_{*-f f}$ given by the short-dashed red curves. The satellite is $\mathrm{DM}$ dominated $\left(f_{\mathrm{DM}, \mathrm{sat}} \simeq 1\right)$ at large distances, implying that $v_{\mathrm{DM}-\mathrm{ff}}$ and $v_{\text {sat-ff }}$ are initially close together. As the satellite falls inwards and loses its DM halo, it becomes dominated by stars $\left(f_{\mathrm{DM} \text {,sat }} \simeq 0\right)$ and $v_{\text {sat-ff }}$ approaches $v_{*-f f}$. Crucially however, $v_{\text {sat-ff }}$ remains above $v_{*-f f}$; for the models of Sec. II it is even above the gravitational escape velocity $v_{\text {esc }}$ at $d_{\text {seg }}$. The satellite is accelerated by the DM force on its DM halo while falling towards $d_{\text {seg }}$, but once this halo has been removed the DM force cannot help keep the stellar remnant bound to the host galaxy after the first pericenter passage. It should therefore be ejected from the host galaxy, not evolve onto the tightly bound orbit needed to produce stellar tidal streams resembling those of the Sgr dwarf as predicted by KNP09.

Dynamical friction, described by the Chandrasekhar formula $[17,31]$

$$
\frac{d \mathbf{v}_{\mathrm{sat}}}{d t} \simeq-\frac{G^{2} m_{\mathrm{sat}} \rho_{\text {host }}}{v_{\mathrm{sat}}^{2}} \hat{\mathbf{v}}_{\mathrm{sat}},
$$

might help keep the stellar remnant bound by reducing its velocity below the escape velocity. However Eq. (17), readily derived from the Chandrasekhar formula, suggests that dynamical friction will not be significant on an orbital time for a binary system with a mass ratio as small as that between the Sgr dwarf and Milky Way. Increasing the mass of the satellite $m_{\text {sat }}$ can increase the effect of dynamical friction, but introduces problems as well. A higher satellite mass will increase the tidal energy $E_{\text {tid }}$ in Eq. (21), which on its own can unbind tidal debris even in the absence of DM forces. The restoring acceleration $a_{\mathrm{DM} \text {-sat }}$ will also increase with the satellite mass, reducing $d_{\text {seg }}$ and increasing the distance over which the DM force has the chance to accelerate the satellite beyond the escape velocity according to Eq. (22). An $N$-body simulation will be required to determine which of these effects dominates for a particular model.

Just such a simulation was performed in the latest version of KNP09 in response to the concerns expressed in this paper. This simulation has precisely the features necessary to avoid ejecting the stellar remnant of Sgr from the Galactic halo. The extraordinarily massive Sgr dwarf $\left(M_{* \text {, sat }}=4 \times 10^{10} M_{\odot}, M_{\mathrm{DM} \text {, sat }}=2 \times 10^{11} M_{\odot}\right)$ experiences strong dynamical friction according to Eq. (24), and its large tangential velocity $v_{\text {sat }}=v_{\text {esc }}(d=250 \mathrm{kpc})=$ $185 \mathrm{~km} / \mathrm{s}$ allows time for this dynamical friction to remove orbital energy before the first close approach to the Galactic center. This simulation demonstrates a possible scenario in which the Sgr dwarf could arrive on its present orbit in the presence of $\beta \simeq 1 \mathrm{DM}$ forces, however several questions remain. The Sgr dwarf loses more than $99 \%$ of its stellar mass during the course of the simulation. Is this large a contribution to the Galactic stellar halo consistent with observations? Although the Galactic DM halo has now been made of "live" particles to allow for dynamical friction, the Galactic disk is still modeled by a static potential. Can the Galactic disk $\left[M_{\text {disk }}=(4.5 \pm 0.5) \times\right.$ $\left.10^{10} M_{\odot}[17]\right]$ survive an encounter with such a massive satellite, particularly in the presence of DM forces? The simulated Galactic DM halo consists of $2 \times 10^{4}$ particles, each of mass $10^{8} M_{\odot}$. Is this sufficient to resolve the dynamical friction on the Sgr stellar core, which also has a mass $M_{* \text { sat }} \simeq 10^{8} M_{\odot}$ after losing $99 \%$ of its mass to tidal disruption? While this new simulation is intriguing, further simulations and comparisons to observations are needed to confirm the validity of the KNP09 scenario.

An alternative possibility to avoid ejecting the stellar remnant of the satellite is if it was accreted by the host galaxy as part of a larger group of satellites. Many of the Milky Way's brightest satellites appear to lie in a disk, as would naturally occur if these satellites were initially members of a group that was subsequently tidally disrupted in the Galactic halo [32]. Lake and D'Onghia [33] specifically proposed that the Magellanic clouds formed the core of such a group that also included seven other Milky Way satellite galaxies including the Sgr dwarf. However, others have argued that this hypothesized group would have to be more tightly bound than observed dwarf-galaxy associations, and that independent accretion from a common filamentary structure could also explain the observed disk of satellites [34]. If the Sgr dwarf was accreted as the more massive member of a satellite-galaxy binary, it could lose energy to its lighter companion as the binary was tidally disrupted in the Galactic halo [35]. Such a "cosmic ménage à trois" might conceivably leave the Sgr dwarf with a small enough orbital energy that its stellar remnant might remain bound to the Milky Way despite the additional acceleration due to $\beta=1 \mathrm{DM}$ forces.

\section{DISCUSSION}

KNP09 performed a series of simulations that suggested that the symmetric tidal streams of the Sgr dwarf galaxy might be consistent with DM forces comparable in strength to gravity, in contradiction with the claims of KK06. These simulations used static potentials for the Galactic bulge, disk, and DM halo. Such static potentials save computational resources and avoid artificial heating of the tidal streams, making them essential to attempts like those in [14] to compare the observed and simulated velocity dispersions in the tidal streams. However, static potentials do not allow for dynamical friction and therefore the satellite galaxy must begin on its current orbit to reproduce the observed tidal streams. Such a cheat was acceptable in [14] and KK06, but fails in the scenario of KNP09 where the segregation of stars and DM should occur on the satellite's first approach within a distance $d_{\text {seg }}$ of the host galaxy. This first approach will likely occur on a very different orbit from the current one with apocentric distance $d_{\mathrm{ap}} \simeq 80 \mathrm{kpc}$ 
needed to produce the observed Sgr tidal streams. The initial conditions used in the first series of simulations in KNP09, with the satellite stars and DM coincident at $d_{\text {ap }}$ with the same velocity, actually correspond to already unbound stellar and DM distributions moving on widely separated orbits in the Galactic halo that happened to converge at $t=0$ when the simulations begin.

We have considered the first approach of a satellite galaxy to the Galactic center in the presence of large DM forces, and have identified three preconditions for the creation of a purely stellar satellite that can go on to form symmetric tidal streams:

(1) The DM force must be strong enough that differential acceleration in the Galactic halo fully segregates the stars and DM prior to the formation of the stellar tidal streams.

(2) The stellar density in the satellite core must be high enough for it to remain a bound object after being pulled free from its DM halo.

(3) The DM force must not accelerate the satellite beyond the gravitational escape velocity of the host galaxy before the satellite stars are pulled from their DM halo.

The extremely large mass-to-light ratios observed even in the cores of Milky Way satellites are difficult to reconcile with condition 2, and such efficient star formation contradicts our theoretical understanding of the vulnerability of these satellites to reionization and stellar feedback. DM forces strong enough to satisfy condition 1 are likely to be too strong to satisfy condition 3 unless a further epicycle is added to give the Sgr dwarf an initial orbital energy much less than that expected for a newly accreted satellite galaxy. While the scenario proposed in KNP09 for symmetric Sgr tidal streams is perhaps possible for certain initial orbits and galactic models, it requires that several restrictive assumptions be satisfied. Further simulations are needed that self-consistently capture both the segregation of stars and DM and the subsequent development of tidal streams.

Future observations of other Milky Way satellites will also severely constrain the existence of $\beta \simeq 1 \mathrm{DM}$ forces. Once their proper motions have been measured, the pericenters of their orbits can be determined. If these pericenters are too close to the Galactic center, the DM forces they will experience during pericentric passages will be inconsistent with retaining their DM halos in seeming contradiction to the high observed mass-to-light ratios. More sophisticated simulations and observations by upcoming astrometry missions like SIM Lite thus provide a clear path towards closing remaining loopholes in constraints on DM forces.

\section{ACKNOWLEDGMENTS}

We would like to thank Andrew Benson, Marc Kamionkowski, Ariel Keselman, and Jim Peebles for useful discussions. An anonymous referee also provided useful comments. This research was supported by NASA ATP/ BEFS grant NNX07AH06G (PI: Phinney).
[1] J. A. Frieman and B. A. Gradwohl, Phys. Rev. Lett. 67, 2926 (1991).

[2] B. A. Gradwohl and J. A. Frieman, Astrophys. J. 398, 407 (1992).

[3] This definition of $\beta$ is identical to that in [4,5] but differs from that in my earlier papers [6,7]. In my earlier papers $\beta$ was the charge-to-mass ratio for the DM particles instead of the ratio between the DM force itself and gravity. The DM force is proportional to the square of the charge as in electromagnetism. The $\beta$ in this paper is therefore equal to $\beta^{2}$ in my previous papers [6,7].

[4] A. Nusser, S. S. Gubser, and P. J. E. Peebles, Phys. Rev. D 71, 083505 (2005).

[5] J. A. Keselman, A. Nusser, and P. J. E. Peebles, Phys. Rev. D 80, 063517 (2009).

[6] M. Kesden and M. Kamionkowski, Phys. Rev. Lett. 97, 131303 (2006).

[7] M. Kesden and M. Kamionkowski, Phys. Rev. D 74, 083007 (2006).

[8] G. R. Farrar and P. J.E. Peebles, Astrophys. J. 604, 1 (2004).

[9] S. S. Gubser and P. J. E. Peebles, Phys. Rev. D 70, 123510
(2004).

[10] S. S. Gubser and P. J. E. Peebles, Phys. Rev. D 70, 123511 (2004).

[11] R. A. Ibata, G. Gilmore, and M. J. Irwin, Nature (London) 370, 194 (1994).

[12] S. R. Majewski, M. F. Skrutskie, M. D. Weinberg, and J. C. Ostheimer, Astrophys. J. 599, 1082 (2003).

[13] V. Belokurov et al., Astrophys. J. 642, L137 (2006).

[14] D. R. Law, K. V. Johnston, and S. R. Majewski, Astrophys. J. 619, 807 (2005).

[15] J. F. Navarro, C. S. Frenk, and S. D. M. White, Astrophys. J. 462, 563 (1996).

[16] J. F. Navarro, C. S. Frenk, and S. D. M. White, Astrophys. J. 490, 493 (1997).

[17] J. Binney and S. Tremaine, Galactic Dynamics (Princeton University Press, Princeton, NJ, 2008), 2nd ed.

[18] J.S. Bullock, A. V. Kravtsov, and D. H. Weinberg, Astrophys. J. 539, 517 (2000).

[19] L. E. Strigari, J. S. Bullock, M. Kaplinghat, J. D. Simon, M. Geha, B. Willman, and M. G. Walker, Nature (London) 454, 1096 (2008)

[20] M. Fellhauer et al., Mon. Not. R. Astron. Soc. 385, 1095 

(2008).

[21] M. Geha, B. Willman, J. D. Simon, L. E. Strigari, E. N. Kirby, D. R. Law, and J. Strader, Astrophys. J. 692, 1464 (2009).

[22] P. J.E. Peebles (private communication).

[23] V. Belokurov et al., Astrophys. J. 658, 337 (2007).

[24] M. G. Abadi, J. F. Navarro, and M. Steinmetz, Astrophys. J. 691, L63 (2009).

[25] J. R. Gott, Astrophys. J. 201, 296 (1975).

[26] J. A. Fillmore and P. Goldreich, Astrophys. J. 281, 1 (1984).

[27] E. Bertschinger, Astrophys. J. Suppl. Ser. 58, 39 (1985).

[28] S.D. M. White and D. Zaritsky, Astrophys. J. 394, 1 (1992).
[29] D. Zaritsky and S. D. M. White, Astrophys. J. 435, 599 (1994).

[30] L. V. Sales, J. F. Navarro, M. G. Abadi, and M. Steinmetz, Mon. Not. R. Astron. Soc. 379, 1464 (2007).

[31] S. Chandrasekhar, Astrophys. J. 97, 255 (1943).

[32] Y.S. Li and A. Helmi, Mon. Not. R. Astron. Soc. 385, 1365 (2008).

[33] G. Lake and E. D’Onghia, Astrophys. J. 686, L61 (2008).

[34] M. Metz, P. Kroupa, C. Theis, G. Hensler, and H. Jerjen, Astrophys. J. 697, 269 (2009).

[35] L. V. Sales, J. F. Navarro, M. G. Abadi, and M. Steinmetz, Mon. Not. R. Astron. Soc. 379, 1475 (2007). 